Jorge E. Jordán-Vaca; Leonardo G. Ballesteros - López; César A. Guerrero-Velástegui Jonathan D. Zúñiga-Rodríguez

https://doi.org/10.35381/cm.v6i10.117

\title{
Enfoque del marketing político en redes web y las consecuencias de percepción política en los electores
}

\section{Approach of political marketing in web networks and its consequences of political perception in the electors}

\author{
Abordagem do marketing político nas redes e suas conseqüências da percepção \\ política nos eleitores \\ Jorge E. Jordán-Vaca \\ jorgeejordan@uta.edu.ec \\ Universidad Técnica de Ambato \\ Ecuador \\ https://orcid.org/0000-0002-8260-4419
}

Leonardo G. Ballesteros - López

leonardoballesteros@uta.edu.ec

Universidad Técnica de Ambato

Ecuador

https://orcid.org/0000-0003-1014-9872

César A. Guerrero-Velástegui

ca.guerrero@uta.edu.ec

Universidad Técnica de Ambato

Ecuador

https://orcid.org/0000-0001-8482-7205

Jonathan D. Zúñiga-Rodríguez

Jzuniga4006@uta.edu.ec

Universidad Técnica de Ambato

Ecuador

https://orcid.org/0000-0002-2528-8392

Recibido: 07/04/2019

Aprobado: 16/05/2019 


\title{
RESUMEN
}

Mediante un estudio amplio e investigativo, enfocado en diferentes modelos de cobertura de la investigación, como experimentales, bibliográficos, y a la vez métodos de investigación exploratoria, descriptiva y cualitativa, que ayudan generar un conocimiento ideal de la relevancia que posee el enfoque del marketing político dentro de la forma de pensar o la forma de percepción que posee el consumidor o en este caso el elector, que ostentan diferentes orientaciones dentro de las redes web en la provincia de Tungurahua correspondiente a Ecuador. Estos enfoques ayudan a conocer de una perspectiva más amplia, cuales son los aspectos y a la vez las ideas necesarias que deben aplicar los partidos políticos dentro de la provincia, para dar a conocer las diferentes propuestas y así llegar con una ventaja o propuesta que penetre en la mente de los electores en los procesos electorales a los que sean aplicados.

Palabras clave: Marketing político; Forma de percepción; Electores; Redes; La web.

\begin{abstract}
Through a broad and investigative study, focused on different models of research coverage, such as experimental, bibliographic, and at the same time methods of exploratory, descriptive and qualitative research, which help to generate an ideal knowledge of the relevance of the political marketing approach within the way of thinking or the form of perception that the consumer has or in this case the voter, who hold different orientations within the web networks in the province of Tungurahua corresponding to Ecuador. These approaches help to know from a broader perspective, what are the aspects and at the same time the necessary ideas that political parties should apply within the province, to make known the different proposals and thus arrive with an advantage or proposal that penetrates into the minds of the voters in the electoral processes to which they are applied.
\end{abstract}

Key Words: Political marketing; Form of perception; Voters; Networks; The web.

\section{RESUMO}

Através de um estudo abrangente e investigação incidiu sobre diferentes modelos de cobertura de pesquisa como experimentais, bibliográfica, enquanto os métodos de pesquisa exploratória, descritiva e qualitativa, que ajudam a criar um perfeito conhecimento da importância que tem o foco de marketing político dentro do modo de 
pensar ou da forma de percepção que o consumidor possui ou, neste caso, o eleitor, que possui diferentes orientações dentro das redes da web na província de Tungurahua correspondente ao Equador. Estas abordagens ajudar a atender uma perspectiva mais ampla, quais são os aspectos e também as idéias necessárias para ser seguido pelos partidos políticos na província de divulgar as diversas propostas e chegar a uma vantagem ou proposta que penetra as mentes dos eleitores nos processos eleitorais aos quais eles são aplicados.

Palavras chave: Marketing político; Forma de percepção; Eleitores; Redes; A web.

\section{INTRODUCCIÓN}

Mediante las diferentes formas de investigación que aportan datos de manera cuantitativa, cualitativa y exploratoria, dentro de la provincia se pudo determinar que la población se divide en dos sectores de investigación, los cuales van a ser identificados al problema en el que se llevan a cabo. Por ello, se obtiene que la población de los electores los cuales son enfoque de estudio en esta investigación, son el eje fundamental para identificar como desean que se aplique el enfoque del marketing, y a la vez los políticos por otra parte, que desarrollan diferentes planes de apoyo para tener un perfeccionismo a los diferentes sectores de los electores.

Para determinar la población de los electores el (CNE, 2019) determina un distributivo electoral de las elecciones del 2019, para así identificar en qué cantones de la provincia de Tungurahua se debe aplicar el medio de estudio, ya que se pretende determinar el entorno global de la provincia. Es por ello que se identifica que en la Provincia de Tungurahua un total de 459454 personas determinadas en las edades de 16 a 65 años son destinadas a ejercer el voto en la provincia. De las 459,454 personas están distribuidas en 224292 hombres y 235162 mujeres en los padrones electorales. Del total de cantones se puede conocer que existen 88 recintos electorales, los cuales comprenden un total de 1384 juntas receptoras del voto, que son distribuidas por toda la Provincia de Tungurahua. Por ello se da a conocer la distribución por cantones. Es por ello que ayuda a conocer que el enfoque del marketing político en los electores, 
genera maneras de percepción que reciben por medio de las redes web al momento de ejercer los diferentes procesos políticos.

Las diferentes etapas políticas que ha tenido que cumplir la Provincia de Tungurahua para ser reconocida como una de las principales ciudades con aporte de personal enfocado en política, genera que los diferentes partidos políticos posean con sus respectivos letreros, contaminar tanto visual como emocionalmente los diferentes espacios públicos que son ocupados por las personas y a la vez estos no generan que los principales políticos no usen espacios tecnológicos como entornos web y redes sociales que son el uso más amplio en la actualidad y sin utilizar espacios públicos.

En la actualidad el marketing político en las redes web, son indispensables para las campañas políticas, ya que es forzoso tener a las redes sociales como una estrategia de comunicación con las personas. Además, al trabajar con los entornos web estos ayudan a influir en los medios convencionales, llegando así a ocupar un papel de infiltrado que genera que las campañas políticas posean una buena comunicación, para entender como la población vive y saber los principales problemas para desarrollar así estrategias en función de las redes web y los comentarios empíricos de los asesores, siendo indispensable el uso de las herramientas web como apoyo a masificar el mercadeo político, (Vargas Marín, 2019), sostiene que la implementación de las redes sociales contribuye a la implementación de acercar la comunicación a las personas para contribuir al logro de metas comunes.

\section{Enfoque del Marketing Político}

Principalmente el enfoque del marketing político, ayuda que los diferentes sectores sean ejes de aplicabilidad de propuestas en las campañas políticas, ya sean por los electores, los cuales sean los que identifiquen porque canales se desarrollen las estrategias, y a la vez que ayuden a generar una ventaja competitiva entre los candidatos o partidos políticos (Fara, 2013). Es por ello que el marketing político, en términos fáciles son todos los objetivos a alcanzar de manera cualitativa o cuantitativa 
de una propuesta, es decir, muchas de las campañas políticas se basan en diferentes estrategias, técnicas que impliquen al marketing para asegurar un éxito, al momento de realizar la campaña política (Serratore \& Salas, 2016).

El marketing político al generar un enfoque en los electores, diseña una imagen pública de los diversos partidos políticos, que a su vez promueven una relación o interacción ente los consultores que están especializados con las diferentes formas de lucha en las elecciones de los diferentes candidatos (Perina \& Izurieta, 2013). Para (Pérez, 2013) el marketing político se enfoca en los electores, ya que ayuda a que se cumplan las reglas de publicidad que socorren a la implementación de tareas que deben llevarse y desarrollarse para tener en un buen objetivo o éxito dentro de una elección de candidatos, al enfocarse en electores, los partidos políticos como organización deben abocarse a establecer mensajes que capten a las diversas comunidades, entre lo cual hoy día se hace pertinente enfocarse en los grupos pro defensa del ambiente, por cuanto esto permite construir una visión diferente de ciudadanía tal como explican Aldana Zavala \& Colina Ysea (2019), siendo basada en el respeto mutuo.

El marketing político es una actividad humana que principalmente es encaminada a generar, crear, fomentar para luego mantener y administrar las diferentes relaciones de intercambio que generan un beneficio entre grupos específicos de electores, mediante una oferta o propuesta política (Vargas, 2013). A más de ello la principal función del marketing político e persuadir y generar que los ciudadanos elijan un candidato 0 partido político al momento de ejercer el voto, ya que es un sistema heterogéneo que involucra principalmente las teorías, técnicas y métodos prácticos que facilitan la comprensión de las propuestas y aspectos políticos que dejan entrar en vigencia (Sánchez, 2017).

Para (Adell \& Alonso, 2014) principalmente el marketing político ayuda a generar un desarrollo de propuestas que sean eje de recolección de información de forma preelectoral y postelectoral, mediante el estudio de un posible posicionamiento, fomentando así la masiva gestión profesional de las diversas campañas políticas. Es 
así que la investigación en la que se desarrolla está enfocada a generar un proceso de campaña que ayude a que los electores generen propuestas principalmente a los candidatos, para así conocer a fondo las necesidades y como desean que se publique los diferentes aspectos que poseen como propuestas de los candidatos (Gan Bustos \& Triginé , 2013). A más de ello se puede determinar que según (Homs, 2014) el marketing político es un paradigma que desarrolla ambientes políticos que llevan al crecimiento de una herramienta que desarrolla procesos amplios y de margen de reconocimiento en las campañas políticas, así mismo esta visión permite estar al frente de organizaciones complejas, (Chirinos Martínez, Perozo Martín, \& Rodríguez Acasio, 2019), exponen la complejización de las empresas, los partidos políticos no pueden estar relegados a una visión tradicional donde solo se enfoquen en captar votos, es necesario que promuevan otros estilos gerenciales para obtener la fidelidad de sus seguidores en la medida que éstos participan activamente en la construcción de los planes sociales.

El marketing Político en términos sencillos y simples en el matrimonio de dos disciplinas de las ciencias sociales, siendo la ciencia política con el mercadeo (Ediratas, Rahayu, \& Natalina, 2013). En una definición de marketing político con mayor número de elementos se puede decir que es la actividad humana encaminada a crear, fomentar, mantener y administrar relaciones de intercambio las mismas que sean beneficiosas entre grupos específicos de electores y una oferta política particular (Vargas, 2013).

\section{Formas de percepción de los electores}

Las formas de percepción de los electores, constituyen parte muy importante de la política del exterior, ya que están principalmente ligadas a la formulación de la contracción de las diferentes naciones en diferentes periodos políticos que se desarrollaron con anterioridad en periodos de campaña electoral (Cabrera , 2014).

La forma de percepción es el aspecto de los objetos al momento de medir la estructura o los elementos que se aplican, ya que forman parte de una representación de una imagen o un estímulo, al oír, escuchar o degustar una percepción subjetiva, temporal o 
a su vez selectiva (Aguillo, 2016). Para (Eskibel, 2015) las diferentes formas de percepción que desarrollan los electores son de percepción visual ya que generan sensaciones que resultan ser estímulos luminosos, coloridos que registran los ojos y a la vez generan señales que se transmiten al cerebro al momento de ver la propuestas de los candidatos.

Dos formas de percepción de los electores al momento de enfocarse en el marketing político de los candidatos, generan estímulos de definición y a la vez tentaciones de realización que ayudan a generar una ventaja competitiva de los otros posibles candidatos (Richard, Caballero, \& Torres, 2015).

La forma de percepción de los electores ha permitido que las campañas políticas modernas se apoyen en dicho medio de comunicación, por el motivo de que les da frecuencia, cobertura, incidencia y permite el posicionamiento en las personas (Medina, 2012), los cual hace uso de las distintas formas de campañas políticas, debido a esto el mensaje que lancen los políticos debe ser con una exactitud que sea capaz de penetrar en la mente del consumidor (Molano, 2014).

Otra forma de percepción del marketing político en los electores ayuda a que el marketing sea un proceso que planea y lleva a cabo determinadas acciones para conseguir satisfacer necesidades a largo plazo, esto mediante la transacción de productos o servicios con el propósito de satisfacer lo que las personas requieren y junto con ella generar beneficios económicos para la empresa (Mesa, 2014).

\section{Percepción electoral}

Para (Villora, Van , \& Lavena, 2012) las percepciones electorales en los electores son perspicacias que ayudan a conocer si los partidos políticos generan causales de corrupción gubernamental, la legitimidad; a la vez los ciudadanos o electores generan percepciones recíprocas, complejas que se evolucionan con el tiempo lo que hace que los electores al momento de ejercer el voto sean más abismados y generen confianza en los políticos. 
La percepción política se considera en muchas de las veces como una nota de conciencia de las personas al poseer las relaciones entre líder del partido, imágenes que genera como marca y a la vez con la exposición y la conciencia que genera el elector al momento por decidir sobre el voto y por consecuencia enfocado en las propuestas (Lock \& Harris , 2013).

La percepción electoral se desarrolla en ambientes de permanecía los cuales dependen mucho de la excelencia del candidato y de las respectivas perspectivas que genera cada uno de los mismos. Los electores como eje funcional se desarrollan bajo estándares que muchas de las veces tienden a ser influencia hacia otras personas, lo cual permite que al interpretar e intervenir con un buen marketing político se pueda enfocar mejores procesos, para así los lectores elegir de mejor manera las propuestas y los desarrollos de los diversos sectores a los que representan.

\section{Implementación de las Redes web}

Las redes web se han desarrollado a tal motivo que llegaron a ser un gran medio de comunicación para la buena difusión de información o datos, los cuales han generado que la información sobre temas políticos ayuden a que se concrete las principales funciones de los políticos y así la vez así poder tener acceso a información sobre temas específicos (Aguillo, 2016).

Las redes web juegan un proceso muy complicado dentro del marketing político ya que posee un correcto manejo de la web, cuando se llega a tener éxito con las diferentes campañas, debido a que el internet permite compartir información de mucha importancia como son propuestas o formas de pensar de los políticos (Sanchez, 2014). El autor (Morales, 2013) que las principales características de las redes web enfocado al marketing político, genera que los candidatos ofrezcan procesos de ventaja competitiva entre los candidatos ya que permite crear una imagen social y a la vez mirar la decisión de la variedad de atributos de presentación al momento de lanzar una campaña política. 
Para culminar con el tema de redes web enfocados al marketing político se obtiene que (Borge, 2005) mira a las redes como una motivación que recoge los diversos datos e información de las opiniones de los electores, los cuales colaboran a corregir, aclarar y mantener los criterios de ideología política que adaptan las capacidades necesarias para alcanzar el objetivo que es el éxito de las diferentes campañas.

\section{METODOLOGÍA}

Como anteriormente se describió en el artículo la investigación que se desarrolla un estudio amplio e investigativo, los cuales poseen modelos de cobertura de la investigación, como experimentales, bibliográficos, exploratorios y a la vez métodos de investigación exploratoria, descriptiva y cualitativa mediante encuestas y opiniones de los electores. A partir de ello para conocer los aspectos más relevantes y conocer de ciencia cierta lo que pide el elector se respalda la información con una encuesta dirigida a todos los cantones de la provincia de Tungurahua.

\section{Investigación Exploratoria}

Para la investigación de la información, una de las herramientas básicas es la técnica de recolección de datos, pues analizaremos antecedentes y criterios de los electores y los políticos quienes ayudarán a obtener datos e información para la investigación, mediante las encuestas que anteriormente fueron explicadas, las que serán utilizadas tanto para electores como para los partidos políticos.

Para la investigación, una de las herramientas más utilizadas es la técnica de recolección de datos, por medio de la cual se puede analizar los antecedentes cualitativos y cuantitativos de los criterios repartidos de los electores y a la vez así se generará una obtención de datos para así tratar de realizar una investigación de campo que ayude a desarrollar procesos de recolección mediante encuesta.

Para obtener datos para reconocer el enfoque del marketing político con la percepción de los electores, se influye en los criterios de los electores tomando encuentra 
CIENCIAMATRIA

Revista Interdisciplinaria de Humanidades, Educación, Ciencia y Tecnología

Año VI. Vol. VI. Nº10. Enero - Julio 2020

Hecho el depósito de ley: pp201602FA4721

ISSN-L: 2542-3029; ISSN: 2610-802X

Universidad Nacional Experimental Francisco de Miranda (UNEFM). Santa Ana de Coro. Venezuela

Jorge E. Jordán-Vaca; Leonardo G. Ballesteros - López; César A. Guerrero-Velástegui Jonathan D. Zúñiga-Rodríguez

principalmente los electores de cada provincia y a la vez de cada sector, los cuales permiten obtener una imagen de como los electores miran a los políticos y cómo influyen los mismos en las campañas políticas y a su vez en los procesos electorales dentro de la provincia. A más de ello con esto se analiza si las personas en una imagen pública de los políticos. Para iniciar principalmente se conoce cuantas personas intervienen dentro del proceso de elecciones, respectivamente en la provincia de Tungurahua, la misma que está distribuida de la siguiente manera según (CNE, 2019).

Tabla 1. Electores Cantón Ambato

\begin{tabular}{|l|l|l|l|l|l|}
\hline Provincia & Cantón & Parroquia & Zona & Juntas & Electores \\
\hline Tungurahua & Ambato & Ambatillo & Ambatillo & 11 & 3601 \\
\hline Tungurahua & Ambato & Ambatillo & Ambatillo alto & 2 & 355 \\
\hline Tungurahua & Ambato & Atahualpa /chipzalata & 25 & 8491 \\
\hline Tungurahua & Ambato & Augusto n Martínez & Augusto n. Martínez & 24 & 7932 \\
\hline Tungurahua & Ambato & Augusto n Martínez & Llatantoma & 2 & 360 \\
\hline Tungurahua & Ambato & Augusto n Martínez & Samanga & 2 & 111 \\
\hline Tungurahua & Ambato & Augusto n Martínez & Laquigo & 2 & 267 \\
\hline Tungurahua & Ambato & Constantino Fernández & 8 & 2483 \\
\hline Tungurahua & Ambato & Cunchibamba & & 10 & 3126 \\
\hline Tungurahua & Ambato & Unamuncho & Unamuncho & 6 & 1763 \\
\hline Tungurahua & Ambato & Unamuncho & La primavera & 2 & 338 \\
\hline Tungurahua & Ambato & Huachi Grande & Huachi Grande & 21 & 6997 \\
\hline Tungurahua & Ambato & Huachi Grande & Huachi San Francisco & 2 & 308 \\
\hline Tungurahua & Ambato & Izamba & & 49 & 17148 \\
\hline Tungurahua & Ambato & Juan Benigno Vela & & 19 & 6504 \\
\hline Tungurahua & Ambato & Montalvo & & 8 & 2987 \\
\hline Tungurahua & Ambato & Pasa & Pasa & 18 & 6080 \\
\hline Tungurahua & Ambato & Pasa & Mogato & 2 & 425 \\
\hline Tungurahua & Ambato & Picayhua & Picayhua & 38 & 12926 \\
\hline Tungurahua & Ambato & Picayhua & Sigsipamba & 2 & 100 \\
\hline Tungurahua & Ambato & Pilahuin & Pilahuin & 23 & 7860 \\
\hline Tungurahua & Ambato & Pilahuin & Tambo loma & 4 & 1155 \\
\hline Tungurahua & Ambato & Pilahuin & El salado & 3 & 807 \\
\hline Tungurahua & Ambato & Quisapincha & Quisapincha & 30 & 10313 \\
\hline Tungurahua & Ambato & Quisapincha & Illagua chico & 3 & 854 \\
\hline Tungurahua & Ambato & San Bartolomé & San Bartolomé & 19 & 6484 \\
\hline Tungurahua & Ambato & San Bartolomé & Angamarquillo & 4 & 956 \\
\hline Tungurahua & Ambato & San Fernando & & 7 & 2215 \\
\hline & & & & 2 \\
\hline
\end{tabular}


CIENCIAMATRIA

Revista Interdisciplinaria de Humanidades, Educación, Ciencia y Tecnología

Año VI. Vol. VI. N¹0. Enero - Julio 2020

Hecho el depósito de ley: pp201602FA4721

ISSN-L: 2542-3029; ISSN: 2610-802X

Universidad Nacional Experimental Francisco de Miranda (UNEFM). Santa Ana de Coro. Venezuela

Jorge E. Jordán-Vaca; Leonardo G. Ballesteros - López; César A. Guerrero-Velástegui Jonathan D. Zúñiga-Rodríguez

\begin{tabular}{|l|l|l|l|l|l|}
\hline Tungurahua & Ambato & Santa Rosa & Santa Rosa & 43 & 14633 \\
\hline Tungurahua & Ambato & Santa Rosa & Cuatro esquinas & 2 & 152 \\
\hline Tungurahua & Ambato & Totoras & Totoras & 14 & 4955 \\
\hline Tungurahua & Ambato & Totoras & Huachi Totoras & 2 & 670 \\
\hline Tungurahua & Ambato & Atocha Ficoa & Atocha Ficoa & 37 & 12943 \\
\hline Tungurahua & Ambato & Atocha Ficoa & Atocha & 2 & 52 \\
\hline Tungurahua & Ambato & Celiano Monge & & 8 & 2646 \\
\hline Tungurahua & Ambato & Huachi Loreto & & 168 & 58532 \\
\hline Tungurahua & Ambato & Huachi Loreto & Voto en casa 1 & 2 & 16 \\
\hline Tungurahua & Ambato & Huachi Loreto & Voto en casa 2 & 1 & 8 \\
\hline Tungurahua & Ambato & Huachi chico & & 80 & 27756 \\
\hline Tungurahua & Ambato & La Matriz & La Matriz & 90 & 31479 \\
\hline Tungurahua & Ambato & La Matriz & Miraflores & 2 & 100 \\
\hline Tungurahua & Ambato & La Merced & & 68 & 23562 \\
\hline Tungurahua & Ambato & La península & & 6 & 1897 \\
\hline Tungurahua & Ambato & Pishilata & Pishilata & 2 & 654 \\
\hline Tungurahua & Ambato & Pishilata & San Vicente & 3 & 853 \\
\hline Tungurahua & Ambato & Pishilata & La joya & 4 & 1130 \\
\hline Tungurahua & Ambato & Pishilata & Techo propio - tiugua & 2 & 221 \\
\hline Tungurahua & Ambato & San Francisco & & 23 & 7923 \\
\hline & & & & \\
\hline
\end{tabular}

Fuente: CNE (2019)

Elaborado: Elaboración propia

*Ambato al ser la ciudad más grande de la provincia de Tungurahua posee un total de 303.041 electores distribuidos en 48 recintos electorales, con un total de 905 juntas receptoras del voto distribuidas en todo el cantón.

Tabla 2. Electores Cantón Baños de Agua Santa

\begin{tabular}{|l|l|l|l|l|l|}
\hline Provincia & Cantón & Parroquia & Zona & Juntas & Electores \\
\hline Tungurahua & Baños & Lligua & & 2 & 438 \\
\hline Tungurahua & Baños & Rio negro & & 4 & 1090 \\
\hline Tungurahua & Baños & Rio verde & & 4 & 1070 \\
\hline Tungurahua & Baños & Ulba & Ulba & 6 & 1570 \\
\hline Tungurahua & Baños & Ulba & Vizcaya & 2 & 139 \\
\hline Tungurahua & Baños & Baños & & 43 & 14782 \\
\hline
\end{tabular}

Fuente: CNE (2019)

Elaborado: Elaboración propia 
*Baños posee un total de 19.089 electores distribuidos en 6 recintos electorales, con un total de 61 juntas receptoras del votos distribuidas en todo el cantón.

Tabla 3. Electores Cantón Cevallos

\begin{tabular}{|l|l|l|l|l|l|}
\hline Provincia & Cantón & Parroquia & Zona & Juntas & Electores \\
\hline Tungurahua & Cevallos & Cevallos & & 24 & 7842 \\
\hline
\end{tabular}

Fuente: CNE (2019)

Elaborado: Elaboración propia

${ }^{*}$ Cevallos posee un total de 7.842 electores distribuidos en 1 recinto electoral, con un total de 24 juntas receptoras del voto distribuidas en todo el cantón.

Tabla 4. Electores Cantón Mocha

\begin{tabular}{|l|l|l|l|l|l|}
\hline Provincia & Cantón & Parroquia & Zona & Juntas & Electores \\
\hline Tungurahua & Mocha & Pinguili & & 4 & 847 \\
\hline Tungurahua & Mocha & Mocha & Mocha & 16 & 5514 \\
\hline Tungurahua & Mocha & Mocha & Yanahurco & 2 & 304 \\
\hline
\end{tabular}

Fuente: CNE (2019)

Elaborado: Elaboración propia

* Mocha posee un total de 6.665 electores distribuidos en 3 recintos electorales, con un total de 22 juntas receptoras del voto distribuidas en todo el cantón.

Tabla 5. Electores Cantón Patate

\begin{tabular}{|l|l|l|l|l|l|}
\hline Provincia & Cantón & Parroquia & Zona & Juntas & Electores \\
\hline Tungurahua & Patate & Los Andes & & 4 & 1036 \\
\hline Tungurahua & Patate & Sucre & & 6 & 1771 \\
\hline Tungurahua & Patate & El triunfo & & 4 & 1123 \\
\hline
\end{tabular}


\begin{tabular}{l|l|l} 
Tungurahua & Patate & Patate
\end{tabular}

Fuente: CNE (2019)

Elaborado: Elaboración propia

* Patate posee un total de 11.598 electores distribuidos en 4 recintos electorales, con un total de 36 juntas receptoras del voto distribuidas en todo el cantón.

Tabla 6. Electores Cantón Quero

\begin{tabular}{|l|l|l|l|l|l|}
\hline Provincia & Cantón & Parroquia & Zona & Junta & Electores \\
\hline Tungurahua & Quero & Yanayacu mochapata & & 4 & 1177 \\
\hline Tungurahua & Quero & Rumipamba & & 4 & 1239 \\
\hline Tungurahua & Quero & Quero & & 42 & 14694 \\
\hline
\end{tabular}

Fuente: CNE (2019)

Elaborado: Elaboración propia

*Quero posee un total de 17.110 electores distribuidos en 3 recintos electorales, con un total de 50 juntas receptoras del voto distribuidas en todo el cantón.

\section{Tabla 7. Electores Cantón San Pedro de Pelileo}

\begin{tabular}{|l|l|l|l|l|l|}
\hline Provincia & Cantón & Parroquia & Zona & Juntas & Electores \\
\hline Tungurahua & Pelileo & Benítez /pachanlica & & 6 & 2024 \\
\hline Tungurahua & Pelileo & Bolívar & & 6 & 2125 \\
\hline Tungurahua & Pelileo & Cotaló & & 6 & 1742 \\
\hline Tungurahua & Pelileo & Chiquicha & 6 & 1841 \\
\hline Tungurahua & Pelileo & El Rosario/rumichaca & & 8 & 2281 \\
\hline Tungurahua & Pelileo & García Moreno & & 9 & 2906 \\
\hline Tungurahua & Pelileo & Huambaló & & 20 & 6516 \\
\hline Tungurahua & Pelileo & Salasaca & & 14 & 4601 \\
\hline Tungurahua & Pelileo & Pelileo & & 66 & 22918 \\
\hline Tungurahua & Pelileo & Pelileo & Voto en & 2 & 9 \\
\hline Tungurahua & Pelileo & Pelileo Grande /r. Mino & & 4 & 989 \\
\hline
\end{tabular}

Fuente: CNE (2019)

Elaborado: Elaboración propia 
*Pelileo posee un total de 47.952 electores distribuidos en 11 recintos electorales, con un total de 147 juntas receptoras del voto distribuidas en todo el cantón.

\section{Tabla 8. Electores Cantón Santiago de Píllaro}

\begin{tabular}{|l|l|l|l|l|l|}
\hline Provincia & Cantón & Parroquia & Zona & Juntas & Electores \\
\hline Tungurahua & Píllaro & Baquerizo Moreno & & 2 & 326 \\
\hline Tungurahua & Píllaro & Emilio María Terán & & 4 & 1243 \\
\hline Tungurahua & Píllaro & Marcos Espinel & & 6 & 1893 \\
\hline Tungurahua & Píllaro & Presidente Urbina & & 4 & 1431 \\
\hline Tungurahua & Píllaro & San Andrés & San Andrés & 28 & 9541 \\
\hline Tungurahua & Píllaro & San Andrés & Huapante Grande & 2 & 338 \\
\hline Tungurahua & Píllaro & San José de poalo & & 6 & 1989 \\
\hline Tungurahua & Píllaro & San miguelito & & 13 & 4351 \\
\hline Tungurahua & Píllaro & Ciudad nueva & & 4 & 1364 \\
\hline Tungurahua & Píllaro & Píllaro & 40 & 13682 \\
\hline
\end{tabular}

Fuente: CNE (2019)

Elaborado: Elaboración propia

*Píllaro posee un total de 36.657 electores distribuidos en 10 recintos electorales, con un total de 109 juntas receptoras del voto distribuidas en todo el cantón.

\section{Tabla 9. Electores Cantón Tisaleo}

\begin{tabular}{|l|l|l|l|l|l|}
\hline Provincia & Cantón & Parroquia & Zona & Juntas & Electores \\
\hline Tungurahua & Tisaleo & Quinchicoto & & 3 & 825 \\
\hline Tungurahua & Tisaleo & Tisaleo & & 27 & 9174 \\
\hline
\end{tabular}

Fuente: CNE (2019)

Elaborado: Elaboración propia

*Tisaleo posee un total de 9.999 electores distribuidos en 2 recintos electorales, con un total de 30 juntas receptoras del voto distribuidas en todo el cantón.

Una vez determinadas todas las bases de datos de los electores, se tiende a 
representar mediante una muestra a todos los sectores de investigación tomando en cuenta que cada uno de los cantones representan un índice ya que, mayoritariamente las ciudades poseen en mayor o menor cantidad de ciudadanos. Por ello se obtiene que un modelo de identificación o de diversificación se basa en un porcentaje claro de representación de los cantones dentro de la provincia de Tungurahua.

Tabla 10. Representación Cantonal

\begin{tabular}{|l|l|l|}
\hline Provincia & Cantón & Representación \\
\hline Tungurahua & Ambato & $65 \%$ \\
\hline Tungurahua & Baños de Agua Santa & $4 \%$ \\
\hline Tungurahua & Cevallos & $2 \%$ \\
\hline Tungurahua & Mocha & $1 \%$ \\
\hline Tungurahua & Patate & $3 \%$ \\
\hline Tungurahua & Pelileo & $11 \%$ \\
\hline Tungurahua & Pílaro & $8 \%$ \\
\hline Tungurahua & Quero & $4 \%$ \\
\hline Tungurahua & Tisaleo & $2 \%$ \\
\hline
\end{tabular}

Elaborado: Elaboración propia

*Se representa con porcentaje la capacidad de representación de los partidos políticos en cada uno de los cantones de la provincia de Tungurahua, teniendo como su principal capital Ambato como una de las principales ciudades de representación.

Partiendo de ello en un amplio margen de confianza en la página de datos (CNE, 2019) se identifica que en la Provincia de Tungurahua un total de 459454 personas entre las edades de 16 a 65 años son destinadas a ejercer el voto en la provincia, de las cuales se tomará el $100 \%$ para un cálculo de encuestas más amplio y preciso para la investigación. Se puede determinar que se va a usar la fórmula de población finita, ya que la variable eludible es mayor a 100000, siendo la siguiente para aplicar al cálculo, por la cual el valor para interpretar los datos es de 383 formas experimentales enfocadas a encuestas.

A partir de los datos obtenidos y la información la mayor cantidad de personas en la 
provincia de Tungurahua se encuentran en el cantón Ambato un 65\% de representación de personas, reconocida por ser una ciudad que da a conocer al país por llevar a puestos gubernamentales gente de los diferentes partidos políticos. Por consiguiente, tenemos al cantón de San Pedro de Pelileo que comprende un 11\% de representación de electores. A más de ello el cantón de Píllaro se considera representativa ya que posee el tercer puesto con $8 \%$ de representación, la cual por consiguiente se puede identificar que Ambato es una ciudad comercial dentro de las ciudades potenciadas de comercio; Pelileo es una cuidad textil, maderera, agrícola y que produce diversos productos en el sector. Por ultimo Píllaro considerada una ciudad donde la población ha aumentado por las representativas costumbres e hitos históricos que desarrollan progreso en ambientes tanto culturales como de fabricación de productos.

\section{RESULTADO}

La importancia principal de conocer la percepción de los electores, al conocer las propuestas $\mathrm{o}$ al conocer los aspectos políticos que se desarrollan diariamente al poseer procesos electorales, lleva a que los partidos políticos y los candidatos generen diferentes percepciones que son determinadas por las actitudes y a la vez por el nivel de conocimiento que poseen los mismos por el marketing político en las redes web. Es por ello que la percepción principal de los electores cree que un $45,3 \%$ de la población de Tungurahua cree que el marketing político ayuda a generar una buena campaña política en los procesos electorales, generando así nuevas estrategias de apoyo que ayuden a los partidos políticos a desarrollarse en un mejor contorno como se identifica a continuación.

Tabla 11. Percepción de ayuda del marketing político

\begin{tabular}{|l|r|r|r|r|r|}
\hline & $\begin{array}{c}\text { Frecuenci } \\
\text { a }\end{array}$ & $\begin{array}{c}\text { Porcentaj } \\
\text { e }\end{array}$ & $\begin{array}{c}\text { Porcentaj } \\
\text { e válido }\end{array}$ & $\begin{array}{c}\text { Porcentaje } \\
\text { acumulad } \\
\text { o }\end{array}$ \\
\hline Válido & Excelente & 31 & 8,1 & 8,1 & 8,1 \\
\hline
\end{tabular}



Jonathan D. Zúñiga-Rodríguez

\begin{tabular}{|l|l|r|r|r|r|}
\hline Bien & 174 & 45,3 & 45,3 & 53,4 \\
\hline Regular & 131 & 34,1 & 34,1 & 87,5 \\
\hline Malo & 39 & 10,2 & 10,2 & 97,7 \\
\hline Deficiente & 9 & 2,3 & 2,3 & 100,0 \\
\hline Total & 384 & 100,0 & 100,0 & \\
\hline
\end{tabular}

\section{Fuente: Elaboración propia}

*La percepción de ayuda del marketing político, reconoce en que forma los electores reconocen como asiste el marketing político en las redes web, a los candidatos en época de elecciones.

La evidencia de una frecuencia de 174 de un total de 384, es una forma de interpretación clara, de que los procesos de percepción política en los electores generan un buen aporte a los procesos electorales, ya que redundan los identificativos para el desarrollo político. Es de mucha importancia conocer que la ayuda del marketing político en las redes web no potencia de una excelente manera, ya que los partidos políticos todavía funcionan con los procesos rudimentarios y no aplican nuevas estrategias de competitividad, para así utilizar las estrategias de desarrollo político.

Tabla 12. Percepción de aplicabilidad del marketing político en los nuevos proceso electorales

\begin{tabular}{|l|l|r|r|r|r|}
\hline \multicolumn{2}{|c|}{} & Frecuencia & Porcentaje & $\begin{array}{c}\text { Porcentaje } \\
\text { válido }\end{array}$ & $\begin{array}{r}\text { Porcentaje } \\
\text { acumulado }\end{array}$ \\
\hline \multirow{3}{*}{ Válido } & Mucho & 52 & 13,5 & 13,5 & 13,5 \\
\cline { 2 - 6 } & Notable & 165 & 43,0 & 43,0 & 56,5 \\
\cline { 2 - 6 } & Algo & 120 & 31,3 & 31,3 & 87,8 \\
\hline \multirow{2}{*}{ Poco } & 42 & 10,9 & 10,9 & 98,7 \\
\hline & Nada & 5 & 1,3 & 1,3 & 100,0 \\
\hline & Total & 384 & 100,0 & 100,0 & \\
\hline
\end{tabular}

Fuente: Elaboración propia

*En esta tabla se menciona en que forma los electores creen que la aplicabilidad del marketing político en las redes web, ayuda a que los candidatos puedan sobresalir con 
sus propuestas en los nuevos procesos electorales de Ecuador en el 2019.

La percepción de los procesos políticos dentro de la provincia de Tungurahua, evidencian que la aplicabilidad del marketing político en las redes web es notable con un $43 \%$, ya que muchos de los partidos políticos dan a conocer a sus candidatos por medio de redes web como lo está establecido con un $56,8 \%$ que las personas utilizan redes sociales para poder dar a conocer las propuestas y los beneficios si llegasen a votar por su candidato de preferencia. Una de las consecuencias más notables dentro de la aplicabilidad del marketing político en redes web es que las personas están de acuerdo con una frecuencia de investigación de 149 de un total de 384 a que se considere al enfoque del marketing político como una ayuda al medio ambiente y así preservar los recursos en mayor cantidad ahorrando plástico, papel, entre otros y tratando de no contaminar los espacios públicos y privados que pueden llegar a ser un problema muy molestoso al momento de elegir candidato, si así lo otorgara una elección de candidatos en procesos electorales.

Una vez identificadas las dos percepciones políticas con las consecuencias en los lectores se puede determinar que una de las fases muy importantes dentro de esta nueva forma de política, es que las personas o electores tengan conceptos claros y específicos de los que realmente se enfoca el marketing político, para así conocer y comprender si el marketing político utilizado por los candidatos ha tenido un gran impacto y a la vez ha marcado una gran diferencia en la población, así también identificando que la población al no entender y comprender el uso de las redes web en los procesos políticos, se sentirían insatisfechos al no pertenecen a quienes prefieren los medios tradicionales y a su vez también que les incomoda la publicidad de política en su navegación en las redes web. 


\section{DISCUSIÓN}

Mediante el análisis e interpretación de las percepciones políticas que poseen los electores, mediante la implementación del marketing político en las redes web, se llega a realizar la indagación de que el marketing político es una buena perspectiva política identificada por los electores, al ser una prioridad y tratar así de impedir de que se siga contaminando el ambiente cultural, patrimonial y a la vez privado con muchos procesos electorales.

Mediante la planeación de las percepciones de los consumidores se puede llegar a identificar que los electores de la actualidad designados entre 16 a 65 años están siendo invadidos por la tecnología, lo cual ayuda a que los partidos políticos lleguen a penetrar de una mejor manera en la memoria de los votantes con las mejores propuestas y así tratando de conseguir que la percepción del voto sea que sea significativa o la vez sea opcional al momento de poseer un proceso electoral. A más de ello si identificamos la perspectiva global de los electores, se puede identificar que identifican una ventaja competitiva mediante la utilización de estrategias adecuadas y así mostrar un interés al tener que mejorar el marketing político, ya que la mayoría de partidos no generan un mayor alcance en las redes vez y a la vez esto lleva a que se desarrollen de manera inadecuada, lo cual acarrea a que los electores se confundan y no puedan identificar los procesos y la propuestas de los candidatos.

\section{CONCLUSIONES}

Se identificó que los diferentes procesos que lleva el marketing político en las redes web, soporta a que la percepción política de los electores y que una de las consecuencias de que esto tenga un aspecto positivo, es que los electores creen que todos los procesos llevados mediante redes web pueden apoyar al momento de decidir un voto, ya que las propuestas en redes web son más claras y precisas al momento de dar a conocer de una en una las opiniones por los candidatos de los partidos políticos. 
Las principales percepciones que maneja los electores es la de ayuda y aplicabilidad que constan de que, si un marketing político ayuda a conocer los diferentes perfiles de candidatos o conocer los diferentes procesos, su aplicabilidad se va a regir mediante la obtención de las nuevas evidencias que se dan a conocer por medio de los entornos web que están aplicados al marketing político.

En la actualidad existe un buen conocimiento sobre marketing político en la sociedad, ya que se identificó que los procesos del merchandesing, ayudan de una buena medida a que las estrategias den buenos resultados, contribuyendo a que se formen diferentes procesos electorales con estrategias que obtengan una mejor aceptación ciudadana, para así satisfacer las diferentes percepciones políticas en los lectores.

\section{REFERENCIAS CONSULTADAS}

1. Adell, Á., \& Alonso, M. (2014). Marketing Político 2.0. España: PAPF.

2. Aldana Zavala, J., \& Colina Ysea, F. (2019). Marketing verde en la conformación de una ciudadanía planetaria en el ámbito educativo latinoamericano. Revista San Gregorio, 0(31), 150-161. Recuperado de http://revista.sangregorio.edu.ec/index.php/REVISTASANGREGORIO/article/vie $\underline{w} / 972$

3. Aguillo, I. (2016). La web social como nuevo medio de comunicación. Barcelona: UOC.

4. Borge, B. (2005). La participación electrónica: estado de la cuestión. Barcelona: UOC.

5. Cabrera , L. (2014). Percepción y Geopolítica en la relación Chile - Perú. EEUU: Palibrio.

6. CNE. (2019). Elecciones seccionales 2019. Obtenido de http://cne.gob.ec/es/?option=com content\&view=article\&layout=edit\&id=4593

7. Chirinos Martínez, A., Perozo Martín, R., \& Rodríguez Acasio, F. (2019). Imágenes gerenciales de Organizaciones bajo un entorno versátil y competitivo. Revista 
doi:http://dx.doi.org/10.35381/r.k.v4i8.224

8. Ediratas, D., Rahayu, D., \& Natalina, A. (2013). Political Marketing Strategy of Jakarta Governor Election in The 2012s. Procedia - Social and Behavioral Sciences, 584 - 588.

9. Eskibel, D. (2015). Psicología para cambiar la política. España: Maquiavelo\&Freud.

10. Fara, C. (2013). Acciones para una buena comunicación en campañas electorales. Buenos Aires: Konrad Adenauer Stiftung.

11. Gan Bustos, F., \& Triginé , J. (2013). Cultura de empresa y gestión de cambios. Madrid: Diaz de Santo.

12. Homs, R. (2014). Marketing para el liderazgo político y social. España: Grijalbo.

13.Lock, A., \& Harris , P. (2013). Political Marketing - vive la différence! Emeraldinsight, 14.

14. Medina, G. (2012). El carisma empaque del candidato. Obtenido de http://www.germanmedinaolarte.com/german-medina-olarte/

15. Mesa, M. (2014). Fundamentos del Marketing. Bogotá: Eco ediciones.

16. Molano, M. (2014). Historia, Experiencia y modernidad de Benjamin. Scielo, 168.

17. Morales, M. (2013). Analítica web para partidos políticos. Barcelona: UOC.

18. Pérez, C. (2013). Comunicación y marketing Político. Bogotá: Legis.

19. Perina, R., \& Izurieta, R. (2013). Estrategias de cominicación para gobernar. Ecuador.

20. Richard, E., Caballero, C., \& Torres, J. (2015). Manual de marketing político. Cómo construir una campaña electoral exitosa. Colombia: Externado.

21. Sánchez, C. (2017). Manual de Marketing Político. Colombia: Ediciones USTA.

22. Sanchez, J. (2014). Manual de uso de redes sociales. Caldas: Universidad de Caldas. 
23. Serratore, N., \& Salas, E. (2016). Análisis de herramientas de marketing politico en campañas presidenciales de Ecuador. Samborondon: Ecuador.

24. Vargas, C. S. (2013). La etnografía en los estudios de marca: una revisión bibliográfica. Pensamiento, 211-234.

25. Vargas Marín, R. (2019). APLICACIÓN DEL INTERNET Y LAS REDES SOCIALES COMO MEDIOS EDUCATIVOS EN EL PROCESO DE ENSEÑANZA. CIENCIAMATRIA, 1(1), 11-23. Recuperado a partir de http://www.cienciamatriarevista.org.ve/index.php/cm/article/view/58

26. Villora, M., Van , G., \& Lavena, C. (2012). Social and Political Consequences of Administrative Corruption: A Study of Public Perceptions in Spain. Wiley Online Library, 73.

\section{REFERENCES CONSULTED}

1. Adell, Á., \& Alonso, M. (2014). Political Marketing 2.0. Spain: PAPF.

2. Aldana Zavala, J., \& Colina Ysea, F. (2019). Green marketing in the conformation of a planetary citizenship in the Latin American educational field. San Gregorio Magazine, $\quad 0 \quad$ (31), 150-161. Recovered from http://revista.sangregorio.edu.ec/index.php/REVISTASANGREGORIO/article/vie w/972

3. Aguillo, I. (2016). The social web as a new means of communication. Barcelona: UOC.

4. Borge, B. (2005). Electronic participation: state of the matter. Barcelona: UOC.

5. Cabrera, L. (2014). Perception and Geopolitics in the Chile - Peru relationship. USA:

Palibrio.

6. CNE (2019). Sectional elections 2019. Retrieved from http://cne.gob.ec/es/?option=com_content\&view=article\&layout=edit\&id=4593

7. Chirinos Martínez, A., Perozo Martín, R., \& Rodríguez Acasio, F. (2019). Management images of Organizations under a versatile and competitive environment. Interdisciplinary Arbitrated Review Koinonía, 4 (8), 4-23. doi: http: //dx.doi.org/10.35381/r.k.v4i8.224 
8. Ediratas, D., Rahayu, D., \& Natalina, A. (2013). Political Marketing Strategy of Jakarta Governor Election in The 2012s. Procedia - Social and Behavioral Sciences,

584-588.

9. Eskibel, D. (2015). Psychology to change politics. Spain: Machiavelli \& Freud.

10. Fara, C. (2013). Actions for good communication in electoral campaigns. Buenos Aires: Konrad

Adenauer Stiftung.

11. Gan Bustos, F., \& Triginé, J. (2013). Company culture and change management. Madrid: Diaz de Santo.

12. Homs, R. (2014). Marketing for political and social leadership. Spain: Grijalbo.

13.Lock, A., \& Harris, P. (2013). Political Marketing - live the différence! Emeraldinsight, 14.

14. Medina, G. (2012). The charisma packing of the candidate. Obtained from http://www.germanmedinaolarte.com/german-medina-olarte/

15. Mesa, M. (2014). Fundamentals of Marketing. Bogotá: Eco editions. 16. Molano, M. (2014). History, Experience and Modernity of Benjamin. Scielo, 168. 17. Morales, M. (2013). Web analytics for political parties. Barcelona: UOC. 18. Pérez, C. (2013). Communication and political marketing. Bogotá: Legis. 19.Perina, R., \& Izurieta, R. (2013). Communication strategies to govern. Ecuador.

20. Richard, E., Caballero, C., \& Torres, J. (2015). Manual of political marketing. How to build a successful election campaign. Colombia: Externado.

21. Sánchez, C. (2017). Political Marketing Manual. Colombia: USTA editions.

22. Sanchez, J. (2014). Manual of use of social networks. Caldas: University of Caldas.

23. Serratore, N., \& Salas, E. (2016). Analysis of political marketing tools in presidential campaigns of Ecuador. Samborondon: Ecuador. 
24. Vargas, C. S. (2013). Ethnography in brand studies: a bibliographic review. Thought,

25. Vargas Marín, R. (2019). APPLICATION OF THE INTERNET AND SOCIAL NETWORKS AS EDUCATIONAL MEANS IN THE TEACHING PROCESS. SCIENCE, 1 (1), 11-23. Recovered from http://www.cienciamatriarevista.org.ve/index.php/cm/article/view/58

26. Villora, M., Van, G., \& Lavena, C. (2012). Social and Political Consequences of Administrative Corruption: A Study of Public Perceptions in Spain. Wiley Online Library, 73.

\section{REFERÊNCIAS CONSULTADAS}

1. Adell, Á. \& Alonso, M. (2014). Marketing Político 2.0. Espanha: PAPF.

2. Aldana Zavala, J., \& Colina Ysea, F. (2019). Marketing verde na conformação de uma cidadania planetária no campo educacional latino-americano. Revista San Gregorio, $\quad 0 \quad$ (31), 150-161. Recuperado de http://revista.sangregorio.edu.ec/index.php/REVISTASANGREGORIO/article/vie w/972

3. Aguillo, I. (2016). A web social como um novo meio de comunicação. Barcelona: UOC.

4. Borge, B. (2005). Participação eletrônica: estado da questão. Barcelona: UOC.

5. Cabrera, L. (2014). Percepção e Geopolítica na relação Chile - Peru. EUA: Palibrio.

6. CNE (2019). Eleições secionais de 2019. Obtido em http://cne.gob.ec/es/?option=com_content\&view=article\&layout=edit\&id=4593

7. Chirinos Martínez, A., Perozo Martín, R. e Rodríguez Acasio, F. (2019). Imagens gerenciais de Organizações em um ambiente versátil e competitivo. Revisão Arbitrada Interdisciplinar Koinonía, 4 (8), 4-23. doi: http: //dx.doi.org/10.35381/r.k.v4i8.224

8. Ediratas, D., Rahayu, D. e Natalina, A. (2013). Estratégia de Marketing Político 
da Eleição do Governador de Jacarta nos anos 2012. Procedia - Ciências Sociais e

Comportamentais,

584-588.

9. Eskibel, D. (2015). Psicologia para mudar a política. Espanha: Maquiavel e Freud.

10. Fara, C. (2013). Ações para uma boa comunicação nas campanhas eleitorais. Buenos Aires: Konrad Adenauer Stiftung.

11. Gan Bustos, F., \& Triginé, J. (2013). Cultura da empresa e gerenciamento de mudanças. Madri: Diaz de Santo.

12. Homs, R. (2014). Marketing para liderança política e social. Espanha: Grijalbo.

13. Lock, A., \& Harris, P. (2013). Marketing Político - viva a diferença! Emeraldinsight,

14.

14. Medina, G. (2012). A embalagem de carisma do candidato. Obtido de http://www.germanmedinaolarte.com/german-medina-olarte/

15. Mesa, M. (2014). Fundamentos de Marketing. Bogotá: edições ecológicas.

16. Molano, M. (2014). História, Experiência e Modernidade de Benjamin. Scielo, 168.

17. Morales, M. (2013). Web analytics para partidos políticos. Barcelona: UOC.

18.Pérez, C. (2013). Comunicação e marketing político. Bogotá: Legis.

19. Perina, R., \& Izurieta, R. (2013). Estratégias de comunicação para governar. Equador.

20. Richard, E., Caballero, C. e Torres, J. (2015). Manual de marketing político. Como construir uma campanha eleitoral bem sucedida. Colômbia: Externado.

21. Sánchez, C. (2017). Manual de Marketing Político. Colômbia: edições USTA.

22. Sanchez, J. (2014). Manual de uso de redes sociais. Caldas: Universidade de Caldas.

23. Serratore, N., \& Salas, E. (2016). Análise de ferramentas de marketing político 


\section{CIENCIAMATRIA}

Revista Interdisciplinaria de Humanidades, Educación, Ciencia y Tecnología

Año VI. Vol. VI. No10. Enero - Julio 2020

Hecho el depósito de ley: pp201602FA4721

ISSN-L: 2542-3029; ISSN: 2610-802X

Universidad Nacional Experimental Francisco de Miranda (UNEFM). Santa Ana de Coro. Venezuela

Jorge E. Jordán-Vaca; Leonardo G. Ballesteros - López; César A. Guerrero-Velástegui

Jonathan D. Zúñiga-Rodríguez

nas campanhas presidenciais do Equador. Samborondon: Equador.

24. Vargas, C. S. (2013). Etnografia em estudos de marca: uma revisão bibliográfica. Pensamento,

211-234.

25. Vargas Marín, R. (2019). APLICAÇÃO DA INTERNET E REDES SOCIAIS COMO MEIOS EDUCATIVOS NO PROCESSO DE ENSINO. CIÊNCIA, 1 (1), 11 23. Recuperado de http://www.cienciamatriarevista.org.ve/index.php/cm/article/view/58

26. Villora, M., Van, G. e Lavena, C. (2012). Conseqüências Sociais e Políticas da Corrupção Administrativa: Um Estudo das Percepções Públicas na Espanha. Biblioteca Online Wiley, 73.

(C2020 por los autores. Este artículo es de acceso abierto y distribuido según los términos y condiciones de la licencia Creative Commons Atribución-NoComercial-Compartirlgual 4.0 Internacional (CC BY-NC-SA 4.0) (https://creativecommons.org/licenses/by-nc-sa/4.0/). 